\title{
Modulation of LPS-Stimulated Astroglial Activation by Ginseng Total Saponins
}

\author{
Sokho Kim ${ }^{1}$, Sehwan Shim ${ }^{1}$, Dea-Seung Choi ${ }^{1}$, Jong-Hoon Kim², Young-Bae Kwon ${ }^{3}$, and \\ Jungkee Kwon ${ }^{1^{*}}$ \\ ${ }^{1}$ Department of Laboratory Animal Medicine, Chonbuk National University College of Veterinary Medicine, Jeonju 561-156, Korea \\ ${ }^{2}$ Department of Veterinary Physiology, Chonbuk National University College of Veterinary Medicine, Jeonju 561-156, Korea \\ ${ }^{3}$ Department of Pharmacology, Chonbuk National University School of Medicine, Jeonju 561-756, Korea
}

\begin{abstract}
Ginseng, a traditional medicine in Asian countries, is known to prevent various neuropathologic diseases such as Alzheimer's. Ginseng total saponins (GTS) in particular are one of the most effective ginseng extract compounds for neuroprotection. However, their protective effects on astrocytes are rarely reported. In pathological circumstances, astroglial activation plays a pivotal role in neuroinflammation. Subsequently, neuroinflammation induced by activated astrocytes causes brain damage. The purpose of the present study was to determine the suppressive effects of GTS on astroglial activation in lipopolysaccharide (LPS)stimulated rat primary astrocytes. Astrocytes treated for $24 \mathrm{~h}$ with LPS demonstrated suppressed glial fibrillary acidic protein expression in a dose-dependent manner in the presence of GTS. GTS reduced production of proinflammatory cytokines such as tumor necrosis factor- $\alpha$ and interleukin- $1 \beta$ and inhibited the level of inducible nitric oxide synthase, and cyclooxygenase- 2 in LPS-stimulated astrocytes. Furthermore, GTS suppressed intracellular reactive oxygen species production. These modulations due to GTS may indicate neuroprotective antiinflammatory properties which may in turn be related to improvements in neurological performance.
\end{abstract}

Keywords: Panax ginseng, Ginseng total saponins, Astrocytes, Lipopolysaccharides, Neuroinflammation

\section{INTRODUTION}

Astrocytes, a major cellular component of neuroglia, serve a wide range of adaptive functions in the mammalian central nervous system (CNS). Astrocyte receptors respond to various neuroactive compounds, including neurotransmitters, neuropeptides, growth factors, cytokines, small molecules, and toxins [1].

Neuroinflammation is a complex biological response to stimuli such as oxidative stress, endotoxins, mechanical injury, and neurodegenerative diseases [2]. Neurodegenerative disorders, such as Alzheimer's disease (AD), Parkinson's disease (PD) and stroke are often accompanied by inflammatory events. Until now, most studies in the field of neuroinflammation focused on the role of microglia. However, recent findings have revealed that astrocytes are not only supportive cells for neuronal functions, but also actively take part in the initiation and modulation of immune reactions in the CNS. Astrocytes that become activated go through a process referred to as reactive astrocytosis, a cellular response to CNS injury [3]. Activated astrocytes have important functions related to initiation and regulation of the CNS immune response mediated via the release of proinflammatory cytokines. Astrocytes are an important source of neuroactive substances such as growth factors, eicosanoids,

\footnotetext{
(c) This is an Open Access article distributed under the terms of the Creative Commons Attribution Non-Commercial License (http://creativecommons.org/licenses/by-nc/3.0/) which permits unrestricted non-commercial use, distribution, and reproduction in any medium, provided the original work is properly cited.
}

Received 19 Oct. 2010, Revised 22 Dec. 2010, Accepted 7 Jan. 2011

*Corresponding author

E-mail: jkwon@chonbuk.ac.kr

Tel: +82-63-270-3884, Fax: +82-63-270-3780 
and neurosteroids that may subsequently influence neuronal development, survival, and neurosecretion [4]. Under pathological conditions, astrocytes release a number of proinflammatory cytokines, such as interleukin (IL)- $1 \beta$, IL-6, tumor necrosis factor (TNF)- $\alpha$, and chemokines.

The pharmacological effects of ginseng have been demonstrated in the CNS and in the cardiovascular, endocrine and immune systems [5-8]. Ginseng is thought to possess antineoplastic, antistress, and antioxidant effects $[9,10]$. Its main constituents are saponins, polysaccharides, alkaloids, and polyacetylenes. Saponins in particular are believed to be responsible for most of the actions of ginseng. Despite several reports in the literature detailing the neuroprotective effects of ginseng, the mechanisms underlying these effects at the molecular and cellular level are rarely reported. Moreover, the modulation effects of ginseng on astroglial activation, which plays a crucial role in the interaction of astrocytes with neurons, have also been rarely reported. In this study, we investigated modulation of astroglial activation by ginseng total saponins (GTS) and the molecular mechanisms involved.

\section{MATERIALS AND METHODS}

\section{Primary astrocyte cultures}

A primary mixed culture of astrocytes was prepared from postnatal day 1 Sprague-Dawley rats [11]. Briefly, cortices were isolated in cold minimum essential medium (MEM) without fetal bovine serum (FBS) and mechanically disrupted by pipetting. The cells were then washed and re-suspended in MEM+10\% FBS (Hyclone, Logan, UT, USA). The disrupted cortices were cultured in a $75 \mathrm{~cm}^{2}$ flask. Enriched astrocyte cultures were obtained after shaking to minimize oligodendrocyte and microglial contamination. The astrocytes were grown in Dulbecco's modified Eagle medium (DMEM)+5\% FBS (Hyclone). GTS dissolved in DMEM was added to the culture medium together with lipopolysaccharide (LPS) from Escherichia coli 055:B5 (Sigma, St. Louis, MO, USA). Cultures were kept in an incubator at $37^{\circ} \mathrm{C}, 100 \%$ humidity, and $95 \%$ air $/ 5 \% \mathrm{CO}_{2}$ atmosphere and used for experiments at the first subculture. GTS from Panax ginseng, extracted and purified by the method of Namba et al. [12] was provided from professor Kim (Chonbuk National University, Korea).

\section{Immunocytochemistry}

Cells were fixed in 100\% methanol and permeabilized with $0.1 \%$ Triton $\mathrm{X}-100$ in phosphate buffered saline (PBS). Non-specific antibody binding was blocked by incubation with $1 \%$ bovine serum albumin in PBS. The cells were then incubated simultaneously with polyclonal rabbit anti- glial fibrillary acidic protein (GFAP) 1:5000 (Millipore, Bedford, MA, USA) overnight at $4^{\circ} \mathrm{C}$. The incorporated GFAP was visualized following successive incubation in goat anti-rabbit $\mathrm{IgG}$ conjugated to fluorescein isothiocyanate 1:300 (Molecular Probes, Eugene, OR, USA). To determine the proportion of proliferating cells, the nuclei were labeled with Hoechst stain (Invitrogen, Carlsbad, CA, USA). Most of the cells exhibited GFAP stain (purity $>95 \%$ ).

\section{Reverse transcription polymerase chain reaction}

The mRNA from astrocytes was isolated using a Ribospin (GeneAll, Seoul, Korea) and subjected to reverse transcription (RT) with a first strand cDNA synthesis kit (Maxime RT PreMix; iNtRON, Seongnam, Korea) according to the manufacturer's instructions. Polymerase chain reaction (PCR) was performed with the following primers: TNF- $\alpha, 5$ '-cacgctcttctgtctactga -3 ' and 5'-ggactccgtgatgtctaagt -3'; IL-1 $\beta, 5$ '-aagctctccacctcaatggacag -3' and 5'-ctcaaactccactttggtcttga-3'; glyceraldehyde 3-phosphate dehydrogenase, 5'-tgaaggtcggtgtgaacggat-3' and 5'-catgtaggccatgaggtccaccac-3'. Each sample was amplified using Maxime PCR PreMix. The PCR products were electrophoresed on $1 \%$ agarose gels and detected by ethidium bromide staining.

\section{Western blotting}

Total proteins from rat astrocyte lysates were subjected to sodium dodecyl sulfate polyacrylamide gel electrophoresis using $10 \%$ to $12 \%$ gels and electrophoretically transferred to polyvinylidene difluoride membranes (Bio-Rad Laboratories Inc., Hercules, CA, USA). Membranes were blocked with 5\% non-fat milk in Tris buffered saline (50 mM Tris base, $\mathrm{pH} 7.5,150 \mathrm{mM} \mathrm{NaCl})$ and incubated individually with a 1:1000 dilution of primary antibodies against nitric oxide synthase (iNOS) (Santa Cruz Biotechnology, Santa Cruz, CA, USA) and cyclooxygenase 2 (COX-2) (Cell Signaling Technology, Beverly, MA, USA). The blots were further incubated with peroxidase-conjugated goat anti-rabbit IgG at 1:20,000 (Millipore). The immunoreactions were visualized using SuperSignal West Dura Extended Duration Substrate (Pierce) and analyzed with a Chemilmager (Alpha Innotech, San Leandro, CA, USA).

Intracellular reactive oxygen species production: evaluation with dicholorofluorescin diacetate assay

Astrocytes plated in a 48-well dish were untreated or 
treated with $1 \mu \mathrm{g} / \mathrm{mL}$ LPS in the absence or presence of GTS and incubated for $6 \mathrm{~h}$. After washing with PBS, cells were stained with $10 \mu \mathrm{M}$ dicholorofluorescin diacetate in PBS for 30 min in the dark. Cells were then washed with PBS twice and extracted with $1 \%$ Triton $\mathrm{X}-100$ in PBS for $10 \mathrm{~min}$ at $37^{\circ} \mathrm{C}$. Fluorescence was recorded with an excitation wavelength of $490 \mathrm{~nm}$ and an emission wavelength of $525 \mathrm{~nm}$.

\section{Statistical analysis}

The results are expressed as the mean \pm standard error of the mean. The data was analyzed using a $t$-test and repeated-measures ANOVA followed by a Bonferroni test. A $p$-value $<0.05$ was considered significant.

\section{RESULTS}

\section{Effect of ginseng total saponins on glial fibrillary acidic protein expression}

To examine the effects of GTS on astroglial activation, we performed GFAP immunocytochemistry and Western blot analysis on reagent-treated cells. GFAP is a major cytoskeletal protein in astrocytes. In addition to its role as a structural integrator, GFAP may provide docking sites for various enzymes implicated in the generation of different astrocytic responses. For this reason, increased GFAP-positive cells are considered signs of activated astrocytes. GFAP-positive astrocytes significantly increased in the LPS-treated cells. However, LPS-elevated GFAP expression was significantly decreased by GTS in a dose-dependent manner (Fig. 1A, B).

\section{Effect of GTS on LPS-induced mRNA expression of proinflammatory cytokines, TNF- $\alpha$, and IL-1 $\beta$}

To examine the effect of GTS on TNF- $\alpha$ and IL- $1 \beta$ gene expression at the transcription level, we determined the level of TNF- $\alpha$ and IL- $1 \beta$ mRNA by RT-PCR analysis in the LPS-stimulated astrocytes. LPS-elicited TNF- $\alpha$ and IL- $1 \beta$ mRNA expression was significantly inhibited by GTS in a dose-dependent manner (Fig. 2A, B).

\section{Effect of ginseng total saponins on reactive oxygen species formation}

In the presence of $1 \mu \mathrm{g} / \mathrm{mL}$ LPS for $24 \mathrm{~h}$, the viability of astrocytes was decreased to $70 \%$ of the control sample. In addition, LPS induced a significant overproduction of ROS to $161.3 \%$ ( $^{* *} p<0.001$ when compared with control). The increased ROS production was blocked with GTS treatment to $68 \%$ at $1 \mu \mathrm{g} / \mathrm{mL}$ GTS ${ }^{++} p<0.001$ when compared with LPS treatment) (Fig. 3). Similarly, astrocyte viability recovered up to $93.4 \%$.

\section{Effect of ginseng total saponins on nitric oxide syn- thase and cyclooxygenase 2 production}

LPS-induced inflammatory cytokine production is required for the activation of signaling pathways including iNOS and COX-2. Therefore, we investigated whether GTS might affect levels of iNOS and COX-2 expression. GTS significantly suppressed the protein levels of both iNOS and COX-2 (Fig. 4). These results suggest that transcriptional events are involved in the inhibitory effects of GTS against LPS-elicited iNOS and COX-2 expression. Changes in the amounts of iNOS and COX-2 enzymes
A
$\mathrm{B}$

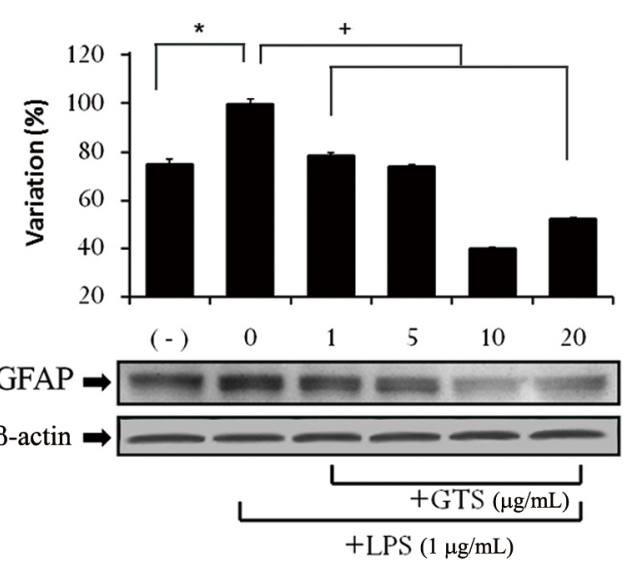

Non-treated control
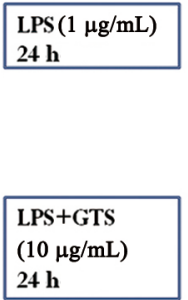
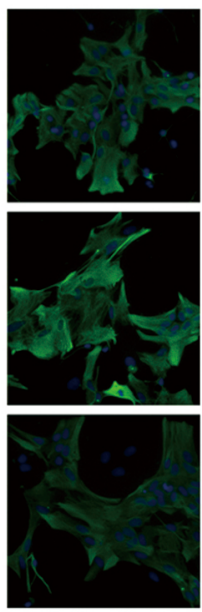

Fig. 1. Inhibitory effects of ginseng total saponins (GTS) on glial fibrillary acidic protein (GFAP) expression. GFAP was determined by Western blot analysis and immunocytochemistry. GFAP is a major cytoskeletal protein in astrocytes. Increased GFAP expression indicates astroglial activation. GTS suppressed GFAP expression in lipopolysaccharide (LPS)-stimulated astrocytes. Data are the mean \pm standard error of the mean of four independent experiments. Statistical analysis: ${ }^{*} p<0.05$ vs. control, ${ }^{+} p<0.05$ vs. LPS $1 \mu \mathrm{g} / \mathrm{mL}$. 
A

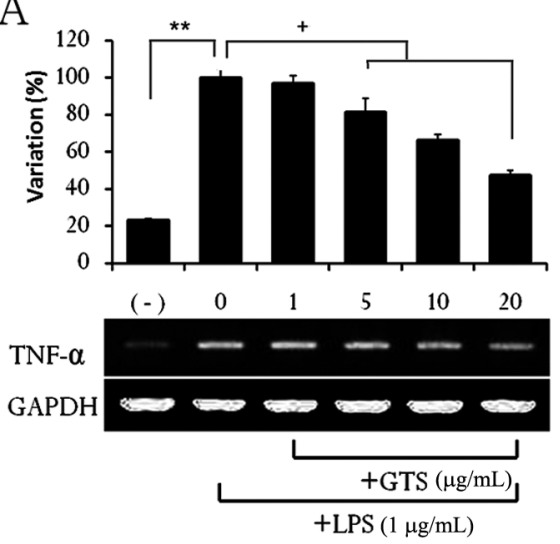

$\mathrm{B}$

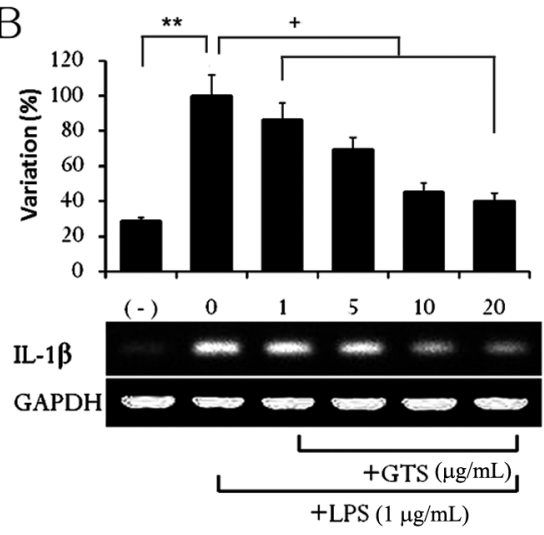

Fig. 2. The effects of ginseng total saponins (GTS) on tumor necrosis factor (TNF)- $\alpha$ and interleukin (IL)-1 $\beta$ in lipopolysaccharide (LPS)-stimulated primary rat astrocytes. Polymerase chain reaction results of the expression of TNF- $\alpha$ and IL-1 $\beta$. LPS induced a robust increase in TNF- $\alpha\left({ }^{* *}, A\right)$ and IL-1 $\beta\left({ }^{* *}, B\right)$ in astrocytes, compared to those without LPS treatment. Rat primary astrocytes were stimulated with LPS for $6 \mathrm{~h}$ in the absence or presence of GTS $(1,5,10$, and $20 \mu \mathrm{g} / \mathrm{mL})$. Data are the mean \pm standard error of the mean of four independent experiments. Statistical analysis: ${ }^{* *} p<0.001$ vs. control, ${ }^{+} p<0.05$ vs. LPS $1 \mu \mathrm{g} / \mathrm{mL}$. GAPDH, glyceraldehyde 3 -phosphate dehydrogenase.

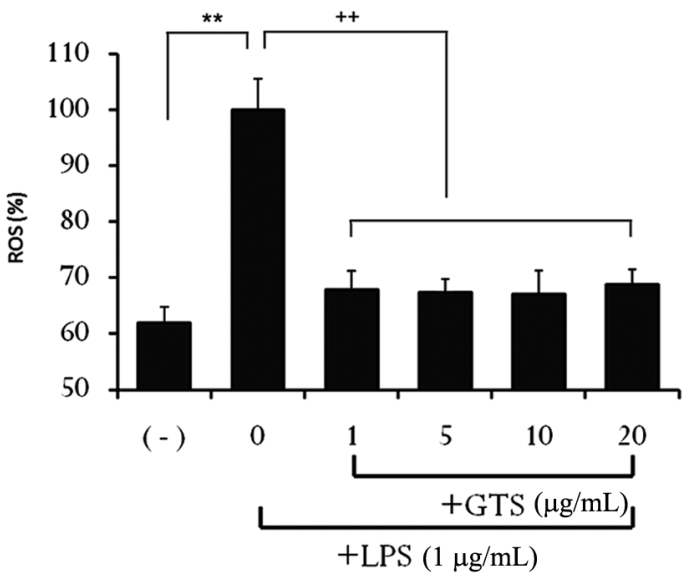

Fig. 3. The reduction effects of ginseng total saponins (GTS) on reactive oxygen species (ROS) production in lipopolysaccharide (LPS)-stimulated primary rat astrocytes. ROS production of primary rat astrocytes treated with LPS $(1 \mu \mathrm{g} / \mathrm{mL})$ in the absence or presence of GTS $(1,5,10$, and $20 \mu \mathrm{g} / \mathrm{mL})$. Data are the mean \pm standard error of the mean of four independent experiments. Statistical analysis: ${ }^{* *} p<0.001$ vs. control, ${ }^{++} p<0.001$ vs. LPS $1 \mu \mathrm{g} / \mathrm{mL}$.

could reflect an alteration in the release of proinflammatory cytokines.

\section{DISCUSSION}

Astrocytes play a crucial role in the homeostasis and function of the CNS, and astrocyte-neuronal interactions secure the survival and normal function of neurons [13]. Several reports in the literature however have indicated that activated astrocytes produce various proinflammatory cytokines and free radicals which play an important role in the processes of neuroinflammatory diseases
$[14,15]$. In the present study, we found that GTS suppressed astroglial activation and its molecular factors. GTS significantly suppressed LPS-induced production of iNOS, COX-2, and ROS in primary astrocytes. RNase protection assays revealed that GTS repressed the expression of proinflammatory cytokines, TNF- $\alpha$ and IL-1 $\beta$, at the mRNA level.

Ginseng is a traditional medicine in Korea, China, and Japan that has been shown to produce a variety of medicinal effects on both the nervous and non-nervous system. Accumulating evidence supports the pharmacological effects of ginseng on the CNS and in the cardiovascular, endocrine, and immune systems as well [5-8]. For example, ginseng components have shown protective effects in experimental models of neurodegeneration [16]. Ginseng appears to act mainly on the hypothalamus and has a sparing action on the adrenal cortex. Indeed, ginseng and its constituents have been thought to possess antineoplastic, antistress, and antioxidant effects $[9,10]$.

The present study was performed to determine whether administration of GTS produces modulation effects on LPS-stimulated astroglial activation. Our results revealed that GTS has strong cytoprotective effects in a dose-dependent manner. LPS-elicited proinflammatory cytokines, TNF- $\alpha$ and IL-1 $1 \beta$, were significantly inhibited by GTS in a dose-dependent manner (Fig. 2). In addition, the IL-1 $\beta$ inhibition by GTS correlated with the suppression of iNOS and COX-2 (Fig. 4), both of which are important mediators in the processes of neurodegeneration [17-19]. A large amount of NO production induced by proinflammatory cytokines plays an important role in neuroinflammation [20]. COX-2 also contributes to 
A

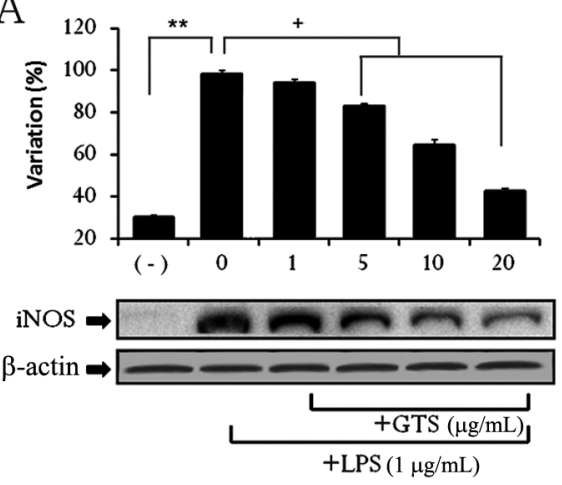

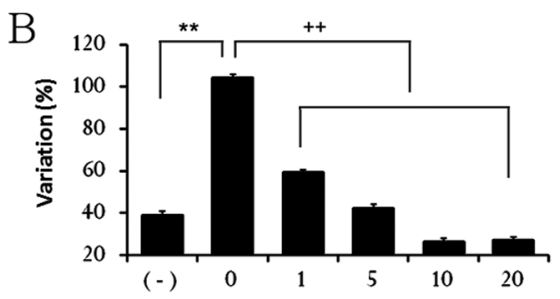

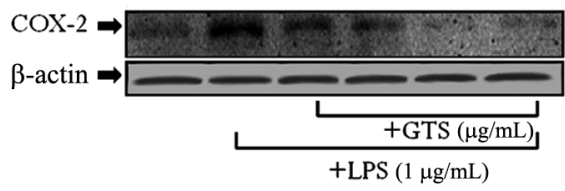

Fig. 4. The effects of ginseng total saponins (GTS) on inflammatory mediators in lipopolysaccharide (LPS)-stimulated primary rat astrocytes. The cells were stimulated with LPS for $24 \mathrm{~h}$ in the absence or presence of GTS $(1,5,10$, and $20 \mu \mathrm{g} / \mathrm{mL})$. LPS as an astrocyte activator significantly induced the expression of inducible nitric oxide synthase (iNOS) and cyclooxygenase 2 (COX-2), which were suppressed by GTS. Data are the mean \pm standard error of the mean of four independent experiments. Statistical analysis: ${ }^{* *} p<0.001 \mathrm{vs.} \mathrm{control,}{ }^{++} p<0.001$ and ${ }^{+} p<0.05$ vs. LPS $1 \mu \mathrm{g} / \mathrm{mL}$.

inflammatory injury in neurodegenerative diseases such as $\mathrm{AD}, \mathrm{PD}$, and stroke [21-25]. Thus, COX-2 functions pathologically in promoting neuronal injury in chronic neuroinflammation [26]. Our studies investigated the antiinflammatory effects of GTS on astrocytes. By detecting major proteins and cytokines related to inflammation, we determined that GTS plays an antiinflammatory role in astrocytes which are a main component of brain glial cells.

In conclusion, the present study showed a neuroprotective effect of GTS on astroglial cells and increased our understanding of several molecular mechanisms involved. Our results suggest the possibility that GTS can be used as a modulating agent to attenuate neuroinflammation and ROS-produced astroglial activation. The strong inhibition of astroglial activation by GTS may provide potential therapeutic strategies for neurodegenerative diseases.

\section{ACKNOWLEDGEMENTS}

This work was supported by a National Research Foundation of Korea Grant funded by the Korean Government (2009-0084511 and 2010-0007257).

\section{REFERENCES}

1. Markiewicz I, Lukomska B. The role of astrocytes in the physiology and pathology of the central nervous system. Acta Neurobiol Exp (Wars) 2006;66:343-358.

2. Cerciat M, Unkila M, Garcia-Segura LM, Arevalo MA. Selective estrogen receptor modulators decrease the production of interleukin- 6 and interferon-gamma-inducible protein-10 by astrocytes exposed to inflammatory chal- lenge in vitro. Glia 2010;58:93-102.

3. Ridet JL, Malhotra SK, Privat A, Gage FH. Reactive astrocytes: cellular and molecular cues to biological function. Trends Neurosci 1997;20:570-577.

4. Liu MH, Lin YS, Sheu SY, Sun JS. Anti-inflammatory effects of daidzein on primary astroglial cell culture. Nutr Neurosci 2009;12:123-134.

5. Tang W, Eisenbrand G. Chinese drugs of plant origin: chemistry, pharmacology, and use in traditional and modern medicine. Berlin: Springer-Verlag, 1992.

6. Attele AS, Wu JA, Yuan CS. Ginseng pharmacology: multiple constituents and multiple actions. Biochem Pharmacol 1999;58:1685-1693.

7. Shah ZA, Gilani RA, Sharma P, Vohora SB. Cerebroprotective effect of Korean ginseng tea against global and focal models of ischemia in rats. J Ethnopharmacol 2005; 101:299-307.

8. Wang LC, Wang B, Ng SY, Lee TF. Effects of ginseng saponins on beta-amyloid-induced amnesia in rats. J Ethnopharmacol 2006;103:103-108.

9. Seong YH, Shin CS, Kim HS, Baba A. Inhibitory effect of ginseng total saponins on glutamate-induced swelling of cultured astrocytes. Biol Pharm Bull 1995;18:1776-1778.

10. Kitts DD, Wijewickreme AN, Hu C. Antioxidant properties of a North American ginseng extract. Mol Cell Biochem 2000;203:1-10.

11. McCarthy KD, de Vellis J. Preparation of separate astroglial and oligodendroglial cell cultures from rat cerebral tissue. J Cell Biol 1980;85:890-902.

12. Namba T, Yoshizaki M, Tominori T, Kobashi K, Mitsui K. Hase J. Fundamental studies on the evaluation of the crude drugs (I). Planta Med 1974;25:28-38.

13. Mehta SL, Manhas N, Raghubir R. Molecular targets in ce- 
rebral ischemia for developing novel therapeutics. Brain Res Rev 2007;54:34-66.

14. Choi C, Park JY, Lee J, Lim JH, Shin EC, Ahn YS, Kim $\mathrm{CH}$, Kim SJ, Kim JD, Choi IS, et al. Fas ligand and Fas are expressed constitutively in human astrocytes and the expression increases with IL-1, IL-6, TNF-alpha, or IFNgamma. J Immunol 1999;162:1889-1895.

15. Lu X, Ma L, Ruan L, Kong Y, Mou H, Zhang Z, Wang Z, Wang JM, Le Y. Resveratrol differentially modulates inflammatory responses of microglia and astrocytes. J Neuroinflammation 2010;7:46.

16. Lian XY, Zhang Z, Stringer JL. Protective effects of ginseng components in a rodent model of neurodegeneration. Ann Neurol 2005;57:642-648.

17. Choi Y, Park SK, Kim HM, Kang JS, Yoon YD, Han SB, Han JW, Yang JS, Han G. Histone deacetylase inhibitor KBH-A42 inhibits cytokine production in RAW 264.7 macrophage cells and in vivo endotoxemia model. Exp Mol Med 2008;40:574-581.

18. Yu Z, Zhang W, Kone BC. Histone deacetylases augment cytokine induction of the iNOS gene. J Am Soc Nephrol 2002;13:2009-2017.

19. Chabane N, Zayed N, Afif H, Mfuna-Endam L, Benderdour M, Boileau C, Martel-Pelletier J, Pelletier JP, Duval N, Fahmi H. Histone deacetylase inhibitors suppress interleukin-1beta-induced nitric oxide and prostaglandin E2 production in human chondrocytes. Osteoarthritis Cartilage 2008; 16:1267-1274.

20. Lockhart BP, Cressey KC, Lepagnol JM. Suppression of nitric oxide formation by tyrosine kinase inhibitors in $\mathrm{mu}-$ rine N9 microglia. Br J Pharmacol 1998;123:879-889.

21. Minghetti L. Cyclooxygenase-2 (COX-2) in inflammatory and degenerative brain diseases. J Neuropathol Exp Neurol 2004;63:901-910.

22. Consilvio C, Vincent AM, Feldman EL. Neuroinflammation, COX-2, and ALS: a dual role? Exp Neurol 2004;187:1-10.

23. Hoozemans JJ, Rozemuller AJ, Janssen I, De Groot CJ, Veerhuis R, Eikelenboom P. Cyclooxygenase expression in microglia and neurons in Alzheimer's disease and control brain. Acta Neuropathol 2001;101:2-8.

24. McGeer PL, McGeer EG. Inflammation and neurodegeneration in Parkinson's disease. Parkinsonism Relat Disord 2004;10 Suppl 1:S3-S7.

25. Teismann P, Vila M, Choi DK, Tieu K, Wu DC, JacksonLewis V, Przedborski S. COX-2 and neurodegeneration in Parkinson's disease. Ann N Y Acad Sci 2003;991:272277.

26. Andreasson K. Emerging roles of PGE2 receptors in models of neurological disease. Prostaglandins Other Lipid Mediat 2010;91:104-112. 